\title{
Eduquer à la santé par la complexité : obstacles et leviers. Apports d'une étude des représentations d'enseignants français et indiens
}

Health education through complexity: obstacles and facilitators. Contribution of a study on the representations of french and indian teachers

\section{France Arboix}

\section{OpenEdition}

Journals

Édition électronique

URL : http://journals.openedition.org/trema/5997

DOI : $10.4000 /$ trema.5997

ISSN : 2107-0997

Éditeur

Faculté d'Éducation de l'université de Montpellier

\section{Référence électronique}

France Arboix, «Eduquer à la santé par la complexité : obstacles et leviers. Apports d'une étude des représentations d'enseignants français et indiens », Tréma [En ligne], 54 | 2020, mis en ligne le 30 novembre 2020, consulté le 14 décembre 2020. URL : http://journals.openedition.org/trema/5997 ; DOI : https://doi.org/10.4000/trema.5997

Ce document a été généré automatiquement le 14 décembre 2020.

Trema 


\section{Eduquer à la santé par la complexité : obstacles et leviers. Apports d'une étude des représentations d'enseignants français et indiens}

Health education through complexity: obstacles and facilitators. Contribution of a study on the representations of french and indian teachers

\section{France Arboix}

\section{Introduction}

1 Préoccupés par la santé des enfants dans un contexte de mondialisation, plusieurs organismes internationaux ont œuvré à développer une politique d'éducation à la santé en milieu scolaire (ES infra) qui permette d'atteindre les objectifs de l' " Education pour tous » (UNESCO, UNICEF, OMS, 2002). L'OMS avait déjà commencé à définir les contours de cette ES dès 1997. Ces préconisations internationales en matière d'ES concernent, entre autres, le contenu de l'ES, sa place dans les Instructions officielles et les responsables de sa mise en œuvre. La théorie de la complexité, telle qu'Edgar Morin l'a formalisée dans La Méthode offre un cadre d'analyse particulièrement opérant pour étudier les enjeux de l'ES. La pensée complexe qu'il s'est attaché à caractériser à travers notamment trois principes directeurs: principe dialogique, récursif et hologrammatique (Morin, 1990) donne une grille de lecture pertinente pour étudier la place et le contenu de l'ES ainsi que le rôle de ses acteurs.

2 Puisque les préconisations internationales s'orientent vers une vision unique, commune, de l'ES, il convient de s'interroger sur les conditions de possibilité de mise en œuvre à l'échelle des particularismes socio-culturels. En effet, si Weaver (1991) 
considère que l'humanité est entrée dans le paradigme de complexité (qu'elle soit désorganisée quand on s'intéresse à a physique quantique; ou organisée quand on s'interesse à l'étude des systèmes), Morin quant à lui considère que la France, marquée par l'empreinte de Descartes, reste bloquée dans un paradigme de simplification propre au monde occidental, qui obère toute conceptualisation de la pensée complexe (Morin, 1990). Dès lors, comment des enseignants issus de contextes socio-culturels divers peuvent-ils mettre en oeuvre une même forme d'ES, définie par les recommandations internationales, alors qu'ils se situent dans des paradigmes différents?

3 Pour apporter certains éléments de réponse à cette question, nous avons défini un cadre d'analyse de l'ES, appuyé sur les principes directeurs de la pensée complexe. Nous avons ensuite opérationnalisé ce cadre pour analyser la politique d'ES préconisée par les experts internationaux. Enfin, nous présentons quelques freins et leviers à la mise en place d'une approche complexe de l'ES dans deux contextes socio-culturels extrêmement différents : l'un français (contexte occidental), l'autre indien (contexte oriental).

\section{Trois formes de pensée pour deux paradigmes}

4 La théorie de la complexité développée par Morin, édicte des principes directeurs permettant d'élaborer une pensée complexe, qui est une alternative au choix binaire entre la pensée simplifiante qui réduit à l'unité et la pensée globalisante (ou holiste) qui réduit au tout. La caractérisation de ces trois formes de pensée (complexe, simplifiante, holiste) font émerger deux paradigmes-maîtres qui offrent un cadre d'analyse à l'ES. C'est ce cadre que nous allons nous attacher à développer dans cette première partie.

\section{Un paradigme de simplification}

5 Le premier grand paradigme, le paradigme de simplification, est caractérisé par la présence d'une pensée simplifiante, héritière des préceptes cartésiens. La pensée simplifiante fait écho à la pensée complexe de Morin. Cette dernière se structure autour de trois piliers (principe dialogique, principe récursif et principe hologrammatique). Nous avons donc cherché les principes de pensée simplifiante que ces principes de complexité permettaient de dépasser.

\section{Pensée simplifiante....}

6 La pensée simplifiante "met de l'ordre dans l'univers et en chasse le désordre " (Morin, 1990), elle se fonde sur la disjonction entre l'esprit et la matière, la philosophie et la science, le corps et l'âme selon un principe de dualité qui oppose les éléments. Le second précepte: "diviser chacune des difficultés que j'examinerai en autant de parcelles qu'il se pourrait, et qu'il serait requis pour mieux les résoudre » (Descartes, 2000, p.49) invite au morcellement du tout en ses parties selon un principe de disjonction. Le troisième précepte : « conduire par ordre mes pensées en commençant par les objets les plus simples et les plus aisés à connaître pour monter peu à peu, comme par degrés, jusqu'à la connaissance des plus composés » (ibid) jette les bases 
d'une pensée linéaire qui interdit les allers-retours, les errances et les tâtonnements permettant de définir un principe de causalité linéaire.

7 Cette pensée simplifiante qui réduit ce qui est multiple à l'unité s'oppose à une pensée globalisante qui réduit ce qui est multiple à la totalité.

\section{.... contre Pensée globalisante ou holiste}

8 Dès le premier tome de La Méthode, Morin considère que c'est dans le cadre de la théorie des systèmes que le holisme aurait émergé pour s'opposer au réductionnisme. Pour lui, cette forme de pensée non-cartésienne semble incapable de distinguer les parties de l'ensemble, de concevoir l'organisation du tout: «le holisme ignore la complexité au sein de l'unité globale» (Morin, 1977, p.124). Cette pensée globalisante ou holiste est en réalité peu développée dans l'œuvre de Morin. Il a d'ailleurs pointé la vacuité de ce concept: "Mais toujours, la totalité n'a été qu'un sac de plastique enveloppant n'importe quoi, n'importe comment et enveloppant trop bien: plus la totalité devenait pleine, plus elle devenait vide.» (Morin, 1990, p.72). Fortin rajoute que le holisme «noie les parties dans une vision homogénéisante du tout. » (Fortin, 2008, p. 37). Il devient alors difficile de trouver des principes directeurs pour structurer cette pensée holiste. Elle reste floue, insaisissable, elle ne permet pas à l'esprit de distinguer et de définir les éléments qui composent l'ensemble : «le tout, dès lors, devient une notion euphorique (puisqu'on ignore les contraintes internes, les pertes de qualité au niveau des parties), fonctionnelle, huilée (puisqu'on ignore les virtualités antagonistes internes), une notion niaise » (Morin, 1977, p.124). Selon l'auteur, la pensée holiste ne pourrait donc pas être décrite ni modélisée, contrairement à la pensée complexe. Nous retiendrons que la pensée holiste ne permet pas à l'esprit de distinguer et de définir. Elle maintient le penseur dans le flou, en perte de repères. D'ailleurs, le terme «holisme» aurait été utilisé pour la première fois par Smuts, homme d'état sudafricain, en 1926 pour « indiquer la tendance de l'univers à construire des unités de complication croissante: matière inerte, matière vivante, matière vivante et pensante $»^{1}$. Selon cette définition, la pensée holiste serait donc également caractérisée par une notion de complication.

9 Ces deux pensées (simplifiante et globalisante) s'opposent sur la façon d'appréhender les phénomènes: l'une ne voit que l'un tandis que l'autre ne voit que le tout sans chercher à comprendre le lien entre les parties et le tout. Ensemble, ces deux pensées forment un grand paradigme de simplification dans lequel règnent la réduction et l'opposition. Lorsque l'on s'intéresse à la question de l'organisation des parties au sein $\mathrm{du}$ tout et sur l'articulation entre le tout et les parties, on rentre alors dans un paradigme de complexité.

\section{Un paradigme de complexité}

La pensée complexe repose sur trois piliers principaux (Morin, 1990), trois principes qui, à partir des principes de la pensée simplifiante, invitent à mettre la pensée en mouvement pour appréhender le réel de façon différente. Parce qu'elle prend ses distances avec les préceptes de Descartes, elle peut être qualifiée de pensée noncartésienne. Les trois piliers de la pensée complexe sont: le principe dialogique, le principe de récursion organisationnelle et le principe hologrammatique. 
11 Il est fondamental de comprendre que la pensée complexe ne s'oppose pas à la pensée simplifiante, sans quoi on retomberait dans le dualisme du paradigme de simplification. Pour se situer dans le paradigme de complexité, il faut réaliser une opération mentale d'intégration de la pensée simplifiante aux principes de pensée complexe.

12 Ainsi au principe dualiste (pensée simplifiante) correspond un principe dialogique (pensée complexe). Le principe dialogique permet d'unir deux éléments antagonistes, deux phénomènes opposés qui devraient se repousser l'un l'autre, mais qui sont complémentaires. Sur certains sujets particulièrement sensibles en ES, comme l'alimentation, il peut arriver que les savoirs véhiculés par l'école rentrent en opposition avec ce qui se passe dans les familles (Jourdan, 2010). Pour éviter à l'élève de se retrouver en situation d'injonction paradoxale, pris entre l'école et sa famille, les acteurs ayant des points de vue divergents, voire opposés, auront intérêt à travailler ensemble, à échanger leur point de vue : à négocier. C'est en effet dans la négociation entre les différents acteurs, qui est au cœur du partenariat (Mérini, 1999) que ces différents savoirs pourront trouver leur juste place dans le débat.

13 Au principe de causalité linéaire (pensée simplifiante) correspond un principe récursif (pensée complexe). Morin(1990) explique que les effets et les causes interagissent dans de nombreuses organisations. Il décrit ce principe comme allant au-delà du principe de la rétroaction (feed-back). Un processus est récursif quand ses produits et effets sont nécessaires à sa propre production et cessation. Ce principe permet de concevoir l'autoproduction et l'auto-organisation, et par là les phénomènes d'émergence. Depuis plusieurs années, les politiques d'éducation à la santé s'orientent vers une ES qui ne se base pas sur une simple vulgarisation des connaissances médicales (Sandrin-Berthon, 1997) supposées modifier le comportement selon un principe de causalité linéaire (transmettre la connaissance biomédicale entraînerait la modification du comportement de santé). Cette nouvelle approche passe par le développement de compétences psychosociales chez les élèves. Nous considérons que le développement des compétences psychosociales constitue une illustration du principe de récursion organisationnelle de la pensée complexe. Les compétences psychosociales visent l'ouverture et l'adaptation de l'individu à son milieu grâce à l'empathie, la gestion du stress, les relations interpersonnelles. Elles permettent également d'exercer son esprit critique sur les connaissances académiques qui, bien que toujours considérées comme nécessaires, apparaissent désormais non suffisantes. Ces compétences s'autoentretiennent et se renforcent au fur et à mesure de leur développement : elles créent une boucle récursive qui dépasse la linéarité du processus de cause à effet. Elles permettent également en retour d'affiner les connaissances sur lesquelles elles s'exercent.

$14 \mathrm{Au}$ principe de disjonction (pensée simplifiante) correspond un principe hologrammatique (pensée complexe). Le principe hologrammatique fait apparaitre le paradoxe de certains systèmes où le tout est inscrit dans chacune des parties singulières qui le constituent. Lorsque l'ES prend à la fois un visage disciplinaire (elle donne naissance à une disciplinaire parmi d'autres) mais qu'on la retrouve aussi à l'intérieur et au carrefour des autres disciplines, elle offre une possibilité d'être travaillée de façon hologrammatique, articulant une vision disciplinaire, à une vision plus large du projet éducatif de l'enfant. C'est dans la convocation de plusieurs disciplines qui vont travailler ensemble et en lien sur des sujets qui touchent à la santé des enfants que l'ES pourra prendre une valeur hologrammatique. Nous touchons ici à 
l'aspect transdisciplinaire de l'ES. Ce terme nécessite une clarification conceptuelle car il peut être parfois utilisé en lien ou de façon interchangeable avec les concepts d'interdisciplinarité, multidisciplinarité, décloisonnement...dans la littérature. Contrairement à la pluridisciplinarité qui permet d'étudier le même objet dans plusieurs disciplines en parallèle, sans jamais se rencontrer, la transdisciplinarité s'appuie sur l'idée de fluctuations des frontières entre les disciplines. Elle s'intéresse à "ce qui est entre ", à «ce qui traverse » et à "ce qui est au-delà » des disciplines (Nicolescu cité par Groussin et Mack, 2013). Cette approche nécessite l'élaboration de schèmes cognitifs qui peuvent traverser les disciplines (Morin, 1994). Cette approche rejoint l'analyse de Lenoir et Sauvé (1998) sur l'interdisciplinarité. Selon ces auteurs, l'interdisciplinarité peut être vue dans une approche dite "ampliative » dépassant les spécificités disciplinaires et favorisant l'émergence d'une nouvelle discipline sur la base d'une carence perçue entre les disciplines existantes.

\section{L'éducation à la santé définie par les préconisations internationales}

Plusieurs textes de cadrage internationaux (UNESCO, UNICEF, OMS, 2002 ; OMS, 1997) ont permis de définir une orientation de l'ES basée sur le partenariat entre différents acteurs (les enseignants, les professionnels de santé, l'ensemble de la communauté éducative et bien sûr les élèves eux-mêmes). De plus, concernant les contenus de l'ES, l'acquisition de connaissances biomédicales relatives à la santé sont considérées comme nécessaires mais non suffisantes. Des compétences psychosociales (OMS, 1993) relatives à l'esprit critique, la résolution de problème, l'assertivité ... apparaissent désormais indispensables à l'exercice d'une responsabilité individuelle et collective en matière de santé. Enfin, concernant la place de l'ES, les experts internationaux considèrent qu'elle devrait donner lieu à la création d'une discipline supplémentaire mais également, se développer à travers les disciplines existantes (OMS, 1997).

Le cadre d'analyse que nous avons défini dans la première partie nous permet de situer cette approche de l'ES dans un paradigme de complexité, comme le synthétise le tableau ${ }^{\circ} 1$. 
Tableau 1 : Grille de lecture pour analyser l'Education à la santé dans le paradigme de complexité

\begin{tabular}{|c|c|c|}
\hline Pensée simplifiante & & Pensée complexe \\
\hline $\begin{array}{l}\text { Principe dualiste } \\
\text { Opposer les points de vue portés } \\
\text { sur un même phénomène } \\
\text { sans jamais les } \\
\text { réunir. }\end{array}$ & $\begin{array}{l}\text { A } \\
C \\
T \\
E \\
\text { U } \\
R \\
S\end{array}$ & $\begin{array}{r}\text { Principe dialogique } \\
\text { Unir deux éléments antagonistes, } \\
\text { deux phénomènes opposés } \\
\text { qui devraient se repousser } \\
\text { l'un l'autre mais qui sont } \\
\text { complémentaires. }\end{array}$ \\
\hline $\begin{array}{l}\text { Peu (ou pas) de collaborations envisagées } \\
\text { Affrontement du contexte formel/non formel }\end{array}$ & & $\begin{array}{l}\text { Les spécificités des acteurs sontreconnues et } \\
\text { mobilisées dans un partenariat (ES définie par } \\
\text { les préconisations internationales) }\end{array}$ \\
\hline Principe de causalité linéaire & $\begin{array}{l}\mathrm{C} \\
\mathrm{O}\end{array}$ & Principe de récursion \\
\hline $\begin{array}{l}\text { Une cause donne une conséquence, } \\
\text { un produit. }\end{array}$ & $\begin{array}{l}\mathrm{N} \\
\mathrm{T} \\
\mathrm{E} \\
\mathrm{N} \\
\mathrm{U}\end{array}$ & $\begin{array}{r}\text { Les causes et les conséquences } \\
\text { interagissent: les produits } \\
\text { deviennent eux-mêmes } \\
\text { producteurs. }\end{array}$ \\
\hline $\begin{array}{l}\text { Transmission de connaissances } \\
\text { biomédicales sur la santé }\end{array}$ & & $\begin{array}{l}\text { Développement de compétences psycho- } \\
\text { sociales en appui sur les connaissances } \\
\text { (ES définie par les préconisations internationales) }\end{array}$ \\
\hline Principe de disjonction & $\begin{array}{l}\mathrm{P} \\
\mathrm{L}\end{array}$ & Principe hologrammatique \\
\hline $\begin{array}{l}\text { Considérer des variables indépendantes } \\
\text { capables d'agir sur l'objet séparé } \\
\text { de son environnement. }\end{array}$ & $\begin{array}{l}\mathrm{A} \\
\mathrm{C} \\
\mathrm{E}\end{array}$ & $\begin{array}{r}\text { Considérer que le tout est inscrit } \\
\text { dans chacune des parties } \\
\text { qui le constituent: } \\
\text { it y a interdépendance } \\
\text { entre le tout et les parties. }\end{array}$ \\
\hline Approche pluri- ou uni-disciplinaire & & $\begin{array}{r}\text { L'ES està la fois une discipline autonome et un } \\
\text { contenu qui traverse les autres disciplines } \\
\text { Approche transdisciplinaire } \\
\text { (ES définie par les préconisations internationales) }\end{array}$ \\
\hline
\end{tabular}

17 Cette politique éducative définie par les experts internationaux (UNESCO, UNICEF, OMS, 2002 ; OMS, 1997), nous permet donc de définir trois critères de complexité en ES : le partenariat entre différents acteurs; l'approche par compétences: le lien entre connaissances et compétences psychosociales; la transdisciplinarité : l'intégration de l'ES dans et à travers toutes les disciplines. Cette politique qui a vocation à être déclinée dans les différents pays à l'échelle mondiale est sous tendue par un paradigme de complexité comme nous venons de le montrer.

18 Pourtant, dans sa réflexion sur les épistémologies du Sud, Santos (2011) soutient que la manière de penser des pays du Nord (eurocentriste) ne permet pas de comprendre les alternatives proposées par d'autres contextes socio-culturels. Il est donc légitime de se demander comment cette politique d'ES est perçue par les enseignants de différents contextes socio-culturels.

19 En France, la montée progressive du modèle des écoles promotrices de santé, inscrite dans l'actuelle stratégie nationale de santé (Ministère des solidarités et de la santé, 2017), traduit une volonté ministérielle de se conformer aux préconisations internationales et d'implémenter cette politique de l'ES complexe dans la politique éducative nationale. Toutefois, Morin (1990) considère que le monde occidental reste encore très marqué par la pensée de Descartes et fonctionne dans un paradigme de simplification où la pensée complexe n'a pas encore sa place.

En Inde, en revanche, le contexte socio-culturel semble plus propice à l'adoption d'un mode de pensée complexe. Ainsi, dans son analyse de la société indienne, Louis Dumont mobilise implicitement plusieurs principes de la pensée complexe (Arboix-Calas, 2013). De plus, dans une analyse de l'œuvre d'Edgar Morin, Grieder (s.d.) défend que l'analyse anthropologique de Morin concernant la civilisation indienne a pu contribuer à la définition de sa théorie de la complexité. Ce serait donc un terrain plus propice au 
développement $\mathrm{du}$ paradigme de complexité. En Inde, les préconisations internationales en matière d'ES sont traduites dans la politique des conseils d'éducation dont dépendent la majorité des établissements scolaires. Les enseignants de notre enquête sont rattachés au $\mathrm{CBSE}^{2}$ qui a publié un manuel d'éducation à la santé toujours en vigueur depuis 2010. Le CBSE traduit assez fidèlement les préconisations internationales en terme de partenariat et de contenu développement de connaissances scientifiques relatives à la santé et de compétences psychosociales. Toutefois en Inde, contrairement à la France, l'éducation à la santé est un champ disciplinaire indépendant, associé à l'éducation physique et sportive.

21 Nous avons cherché à savoir comment les enseignants issus de deux paradigmes si différents, percevaient les trois critères de complexité de l'ES que nous avons définis: Partenariat entre les différents acteurs; Approche compétences: les connaissances s'associent aux compétences psychosociales; Transdisciplinarité : l'ES traverse toutes les disciplines. Nous avons analysé les représentations d'enseignants français et indiens dans une enquête menée de 2011 à 2012.

\section{Freins et leviers à la mise en œuvre de cette ES dans deux contextes socio-culturels différents.}

\section{Cadre méthodologique}

Dans le but d'identifier les freins et leviers au développement d'une ES complexe, définie par les trois critères précédemment identifiés (partenariat, contenu basé sur les connaissances et les compétences, transdisciplinarité) nous avons recueilli les représentations de 98 enseignants français dans l'académie de Montpellier (France) d'avril à octobre 2012 et 93 enseignants indiens dans le territoire de Pondichéry (Inde) de février à avril 2011. Cette enquête s'inscrivait dans le cadre d'une recherche sur la prévention de l'obésité. Les enseignants appartiennent à des écoles primaires, tirées au sort parmi la liste des établissements répertoriés dans chaque contexte (Montpellier, Pondichéry).

De très nombreux auteurs ont proposé des modèles pour l'étude des représentations sociales qui constituent des représentations partagées par un groupe social (Moscovici, 1961 ; Barthes et Alpe, 2016). Nous avons choisi de nous référer au modèle de Moscovici qui propose de distinguer trois éléments dans les représentations: opinion, attitude, stéréotypes. Moscovici considère que ces trois éléments constituent des formes d'organisation de la réponse à une question en vue de l'élaboration d'une conduite (Moscovici, 1961). Les représentations ont donc une influence sur les pratiques. Nous nous sommes inspirés de l'étude menée par Négura (2006) qui propose une analyse de contenu des représentations sociales déclinée en: analyse thématique catégorielle qui donne accès aux opinions; analyse des propositions évaluatives qui donne accès aux attitudes (critiques positives ou négatives) et analyse des stéréotypes. Nous espérons ainsi identifier quelques freins et leviers de la pratique de l'ES.

L'enquête a consisté en un test d'associations de mots (fonctionnant sur un système de stimulus "inducteurs» et de réponses «induites»). Dans le cadre d'une action envisagée sur le thème de l'ES, nous avons présenté les trois critères de complexité aux enseignants afin de recueillir leurs représentations : 
« Listez les 10 termes qui vous viennent à l'esprit lorsque vous entendez :

- Travailler en partenariat : réponse $\mathrm{N}^{\circ} 1$, réponse $\mathrm{N}^{\circ} 2, \ldots . .$. réponse $\mathrm{N}^{\circ} 10$.

- Développer les compétences spécifiques (psychosociales) des élèves : estime de soi, esprit critique, résolution de problèmes... : réponse $\mathrm{N}^{\circ} 1$, réponse $\mathrm{N}^{\circ} 2, \ldots .$. réponse $\mathrm{N}^{\circ} 10$.

- Travailler en transdisciplinarité: aborder l'ES dans et à travers toutes les disciplines: réponse $\mathrm{N}^{\circ} 1$, réponse $\mathrm{N}^{\circ} 2, \ldots .$. .réponse $\mathrm{N}^{\circ} 10$. »

Nous avons ensuite réalisé une analyse thématique en regroupant les termes en champs sémantiques et une analyse des propositions évaluatives en regroupant deux champs sémantiques distincts : critiques positives et critiques négatives. Cette analyse s'est déroulée en deux temps et a été réalisée par trois codeurs différents pour tenter de diminuer la subjectivité de l'interprétation des termes.

Dans un premier temps, nous avons réalisé notre étude de terrain en Inde et commencé par le traitement du corpus indien. Le type de catégories n'était pas donné à l'avance et nous avons opéré par induction. Dans cette " procédure par tas » (Bardin, 2007), le nom de chaque champ lexical est défini en fin de classement. Deux codeurs ont réalisé le classement en aveugle puis un troisième codeur a contribué à valider les champs qui ne faisaient pas l'objet de consensus entre les deux premiers codeurs.

Dans un second temps, nous avons traité le corpus français. Nous avons utilisé la " procédure par boîtes » (ibid) : grâce aux catégories définies dans le classement indien, nous avons réparti les différents termes du corpus français, de la meilleure façon possible, au fur et à mesure de leur rencontre dans le corpus. L'intervention des trois codeurs a suivi la même logique que pour la " procédure par tas ». Une fois l'ensemble des champs sémantiques constitués, nous avons évalué le poids relatif de chaque champ sémantique en calculant le nombre moyen de termes par enseignant.

Pour Bardin (2007), ce type de test permet également de réaliser une analyse des stéréotypes sociaux spontanément partagés par les membres d'un groupe. Ils émergent à travers la répétition des réponses. Nous avons retenu le seuil de $10 \%$ de répétions à l'intérieur de chaque champ. Le stéréotype peut être un frein à la pratique selon qu'on le retrouve significativement plus souvent chez les enseignants qui ne font pas de l'ES dans leur classe ou un catalyseur selon qu'on le retrouve significativement plus souvent chez les enseignants qui font de l'ES dans leur classe. Nous avons évalué si la présence de ces stéréotypes au sein de chaque groupe (pratiquant Es vs non pratiquant ES) était significativement différente avec un test $t$ de comparaison de moyenne non-paramétrique (test de Wilcoxon-Mann-Whitney) grâce au logiciel R.

30 Enfin, l'analyse des représentations renseignera également sur les attitudes des répondants à travers les critiques qu'ils vont formuler. Une attitude est caractérisée par une composante affective. Sa direction est déterminée par le sens qu'elle occupe sur une échelle bipolaire: elle peut être positive ou négative (Bardin, 2007). Une attitude peut donc être favorable, défavorable ou ambivalente (ibid). L'identification de catégories exprimant un jugement sur la proposition (critiques positives ou négatives) signera la présence d'attitudes particulières dans les représentations.

\section{Résultats}

31 L'échantillon français est constitué d'une majorité de femmes (81.5\%) qui se situent plutôt en milieu de carrière (moyenne d'âge : 42 ans) et $78 \%$ de cet échantillon déclare 
aborder l'ES en classe. L'échantillon indien est constitué d'une très grande majorité de femmes (99\%) un peu plus jeunes (moyenne d'âge : 35,2 ans). Seulement 63\% d'entre elles déclarent pratiquer l'ES. Ces données sont représentatives de la population des enseignants du premier degré en France puisque les femmes représentaient $82,2 \%$ en 2013, pour un âge moyen de 41,6ans (Ministère de l'Education nationale, 2014). En Inde, notre échantillon est en revanche un peu au-dessus de la moyenne nationale estimée à 93\% de femmes dans le primaire en 2011 (Ministry of Human Resource Development, 2018).

\section{Présentation des réponses}

En contexte français, les enseignants fournissent un nombre de mots induits assez faibles face à ces trois critères de complexité : 6,55 réponses pour le partenariat ; 5,55 réponses pour l'approche compétences et 4,88 réponses pour la transdisciplinarité. Cela peut signer un manque de recul ou une perplexité face à ces critères. Le poids relatif (taille du champ/nombre de réponses total) des champs sémantiques dans les réponses des enseignants français sont répertoriés dans le tableau $n^{\circ} 2$.

En contexte indien, les enseignants fournissent un nombre de mots induits plus conséquent face à ces trois critères de complexité : 7,67 réponses pour le partenariat ; 8,29 réponses pour l'approche compétences et 8,06 réponses pour la transdisciplinarité. Cela peut signer une certaine aisance du public indien face à ces critères de complexité. Le poids relatif des champs sémantiques dans les réponses des enseignants indiens sont répertoriés dans le tableau $\mathrm{n}^{\circ} 3$.

Les réponses des enseignants sont présentées ci-dessous en distinguant deux groupes : les enseignants pratiquant l'ES et les enseignants non-pratiquant l'ES. L'analyse des réponses dans chaque groupe permettra d'identifier plus finement les freins et leviers à la pratique de l'ES. En outre, une différence importante entre les pratiquants ES et les non pratiquants ES pourrait signer la présence d'obstacles épistémologiques chez ces derniers. 
Tableau 2 : Diagramme des poids relatifs (taille du champ/nombre de réponses total) des champs sémantiques dans les réponses des enseignants français face aux trois critères de complexité

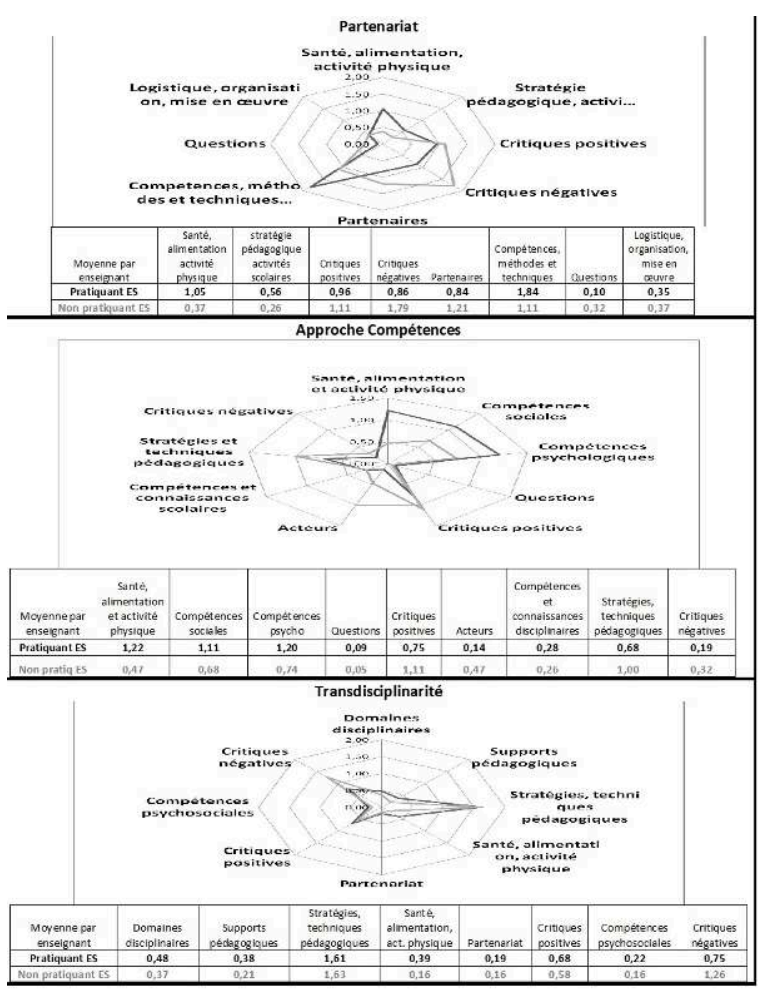

Tableau 3 : Diagramme des poids relatif (taille du champ/nombre de réponses total) des champs sémantiques dans les réponses des enseignants indiens face aux trois critères de complexité

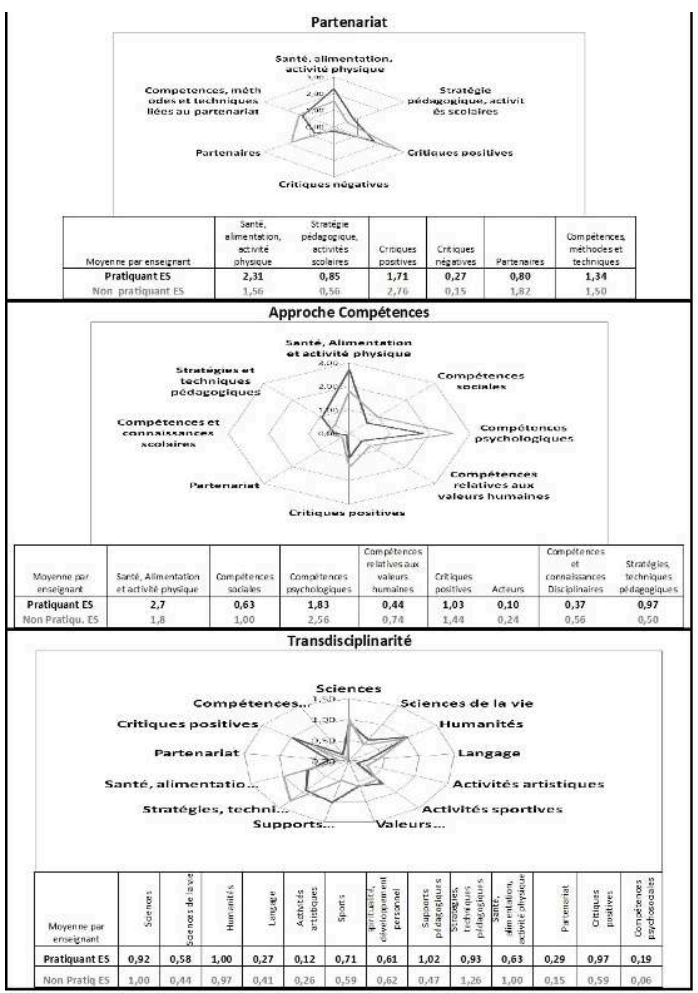




\section{Analyse des réponses}

L'étude des représentations des enseignants à travers l'identification des opinions, attitudes et stéréotypes, nous permet d'identifier des freins et leviers à la mise en œuvre de l'ES prescrite dans les Instructions officielles de chaque contexte (Ministère de l'Education nationale en France et CBSE en Inde).

\section{Partenariat}

\section{Points de convergence}

Les enseignants, qu'ils soient français ou indiens partagent certains éléments dans leurs représentations. Les opinions face au partenariat s'orientent pour une large part vers les compétences, méthodes et techniques à développer. C'est le champ qui a le plus de poids en contexte français. A titre d'exemple, nous pouvons citer dans les réponses des français: "l'échange ", "le partage », "la communication », "le respect » et «l'intérêt de monter des projets ». Elles font écho aux réponses des indiens telles que «sharing", « adjustable with others ", « cooperation » qui montrent une volonté de cohésion et de recherche de consensus.

Les enseignants français et indiens partagent dans leurs représentations un stéréotype de difficulté associé à ce critère de complexité mais il n'est pas significativement relié à une pratique ou une absence effective de pratique de l'ES ( $>00,05)$.

\section{Points de divergence}

8 Les enseignants des deux contextes ont une attitude ambivalente à l'égard du partenariat puisque l'on retrouve des critiques à la fois positives et négatives. Toutefois la part des critiques négatives en contexte indien est extrêmement faible par rapport au contexte français. Les critiques positives, sont au contraire, le champ qui a le plus de poids en contexte indien comparativement aux autres champs. Elles mettent l'accent sur l'épanouissement des enseignants à travers des termes comme "happy", "satisfaction" ou encore sur la pertinence du partenariat "learning new things", « interesting », « useful ».

9 Les réponses des français sont porteuses de plusieurs stéréotypes : trouver le travail en partenariat " enrichissant », «travailler dans le cadre d'un projet » pourraient avoir un effet catalyseur sur les pratiques d'ES (le stéréotype est significativement plus présent chez les enseignants pratiquant l'ES avec $p<0,05$ et $p<0,01$ respectivement). D'autres stéréotypes en relation avec le temps consacré à l'organisation du partenariat sont relevés mais n'ont pas d'effet significatif sur la pratique puisque retrouvés dans les deux groupes pratiquant et non pratiquant l'ES $(p>0,05)$ : «manque de temps", « disponibilité », « trop de travail ».

En contexte indien, les enseignants pratiquant l'ES évoquent beaucoup plus souvent le champ de la santé, alimentation, activité physique mais moins de partenaires de travail que les enseignants non pratiquant. Ces derniers auraient peut-être moins de connaissances dans ces domaines, ce qui pourrait constituer un frein à leur pratique. Cette hypothèse pourrait expliquer qu'ils citent plus de partenaires potentiels pour réaliser ce travail (essentiellement les professionnels de santé). Il est probable que les non pratiquant ES cherchent à pallier ce manque de connaissances en déléguant la 
responsabilité de cette éducation à des professionnels de santé qu'ils jugent mieux formés, ce qui pourrait expliquer cette absence de pratique.

\section{Approche Compétences}

\section{Points de convergence}

Les diagrammes des champs lexicaux montrent que les opinions des enseignants s'orientent vers la pertinence de mettre ces compétences en lien avec les connaissances biomédicales, dans le domaine de l'alimentation et de l'activité physique. Ils situent ces compétences dans le champ psychologique du rapport à soi (compétences psychologiques) et du rapport aux autres (compétences sociales) bien que cela soit plus discret en contexte indien.

\section{Points de divergence}

Les enseignants indiens ont une attitude très favorable vis-à-vis de cette pratique avec des critiques positives qui expriment leur approbation: "good culture", "better future ", " excellent ». En revanche, les français restent dans une attitude ambivalente vis-à-vis de cette pratique (présence simultanée de critiques positives et négatives). Nous avons relevé plusieurs questions : « comment faire? ", « dans quel but?», « quels outils? " qui peuvent traduire la perplexité des enseignants français. En revanche, aucune question ne ressort en contexte indien.

\section{Transdisciplinarité}

\section{Points de convergence}

Dans les deux contextes, les opinions des enseignants se focalisent sur les stratégies et techniques pédagogiques à développer pour pouvoir travailler de façon transdisciplinaire. Ils partagent ici une préoccupation commune liée au cœur de leur métier. Les français citent par exemple : "la nécessiter de faire du lien ", " donner du sens aux apprentissages", "les échanges de service, de connaissance", «l'enrichissement du vocabulaire » mais par-dessus tout, le travail par «projets ». Les réponses de leurs homologues indiens reflètent des stratégies plus concrètes, telles que : « story telling method », « introduction at early class », « playing games ».

Dans les deux contextes, nous avons identifié un stéréotype majeur dans les représentations des enseignants. Chez les français, c'est le montage de projet; chez les indiens, c'est le travail par sensibilisation (awareness). Toutefois, la présence de ces stéréotypes n'est pas significativement différente entre les pratiquants et les non pratiquants l' ES (p>0,05). Ils n'ont donc à priori pas d'effet sur le choix de faire ou non de l'Es.

\section{Points de divergence}

Les enseignants indiens n'émettent pas de critiques négatives sur la transdisciplinarité alors que nous relevons un nombre important de critiques négatives chez les français telles qu'éprouver un sentiment de «difficulté » et évoquer le "manque de temps ». La présence de nombreuses questions: «à quel moment?", «à quel niveau?», 
«comment?», «quels supports? "signent également la perplexité des enseignants français face au défi de la transdisciplinarité.

Contrairement aux enseignants français qui ont cité peu de domaines disciplinaires, les enseignants indiens en citent énormément, si bien que nous les avons répertoriés en champs indépendants: sciences générales, sciences de la vie, humanités, langage, activité artistiques et activités sportives. Cette diversité disciplinaire contraste avec le fait qu'en Inde, l'éducation à la santé est censée être une discipline indépendante qui n'invite pas, à priori, à la transdisciplinarité. Les enseignants indiens établissent donc tout naturellement des liens avec les autres disciplines dans leurs réponses alors que les français ne le font pas.

\section{Discussion}

47 Nous nous étions demandé comment des enseignants issus de contextes socio-culturels différents pouvaient mettre en œuvre une même forme d'ES s'ils se situaient dans des paradigmes différents. Les représentations des enseignants face aux trois critères de complexité de l'ES que sont le travail en partenariat, l'approche par les compétences psychosociales et la transdisciplinarité font apparaitre une certaine convergence indépendamment des paradigmes dans lesquels ils se trouvent mais également certains points de divergence.

Que l'on soit familier ou non de la pensée complexe, le travail en partenariat fait toujours courir le risque d'une délégation de responsabilité. Nous retrouvons ce risque dans certaines réponses stéréotypées des enseignants français sur l'aspect chronophage du partenariat. Toutefois, selon notre étude, le partenariat semble optimisé par le travail en mode projet. Pour Mérini (1999), le partenariat se construit par la négociation en vue de résoudre un problème commun. Les enseignants doivent donc se sentir réellement concernés par la santé de leurs élèves. Pour que le partenariat fonctionne réellement, Bizonni-Prévieux et al. (2011) ont montré, dans une étude sur les enseignants français, que le travail en mode projet ne permet au partenariat de se mettre en place que si c'est l'enseignant qui en est à l'initiative. Dans le cas contraire, celui-ci devient simple délégation ou sous-traitance.

Nous retrouvons également le risque de délégation de responsabilité chez les enseignants indiens. Dans notre étude, nous avons vu que les enseignants non pratiquant l'ES vont citer moins de connaissances biomédicales mais davantage de partenaires qui pourraient donc palier à ce manque de connaissances en se substituant à eux.

50 Nous constatons que les enseignants associent également dans les deux contextes, les connaissances biomédicales aux compétences psychosociales. Cependant, notre étude ne nous permet pas d'identifier s'il y a opposition entre ces deux objets (connaissances et compétences) auquel cas nous serions dans une logique de simplification, ou bien s'il y a une véritable volonté d'articuler et d'intégrer les connaissances aux compétences.

51 Toutefois, la présence de critiques négatives faisant ressortir la notion de "difficulté » uniquement en contexte français suggère que les enseignants français ont du mal à intégrer ces connaissances biomédicales aux compétences. L'assimilation du concept de complexité à celui de difficulté, plusieurs fois souligné dans la littérature (Ardoino cité par Alhadeff-Jones, 2008; Morin, 1988), a été mis au jour dans notre étude. Mais ce stéréotype ne constitue pas pour autant un obstacle pour les enseignants français 
puisqu'il est présent chez les deux groupes d'enseignants (pratiquant et non pratiquant l'ES) sans qu'aucune différence significative n'ait pu être identifiée ( $p>0,05)$.

Nous avons vu dans la première partie que réaliser cette intégration des connaissances aux compétences requiert de passer d'une logique de causalité linéaire à une logique récursive dans laquelle les compétences se développent sur les connaissances qu'elles permettent en retour d'enrichir. Cette opération mentale nécessite de surmonter l'obstacle épistémologique de la dualité qui oppose la primauté des savoirs (dogmatisme) à la primauté des compétences au motif que tous les savoirs auraient la même valeur (relativisme). Cette opposition constitue l'un des principaux pièges des "éducations à " (Fabre, 2014) dans lequel tombent souvent les enseignants peu familiers d'une pensée complexe. En effet, Lange et Victor (2006) considèrent que l'appréhension des objets de savoir académique et des compétences de l'élève sont vécues le plus souvent par les enseignants de façon antagoniste.

D'autre part, l'impression de flou, la perte de repères, traduite par le questionnement des enseignants français face aux contenus des « éducations à » a déjà été identifiée par plusieurs auteurs en contexte français (Fabre, 2014 ; Pizon et al., 2010) mais n'avait pas encore été recherchée en contexte indien, à notre connaissance. La présente étude montre qu'en Inde où le contexte socio-culturel est plus propice à l'adoption d'une pensée complexe, les enseignants n'expriment ni difficultés, ni interrogations face au développement des compétences psychosociales des élèves ou à la transdisciplinarlité de l'ES. Cette impression de flou serait donc absente dans le contexte indien, familier de la pensée complexe mais bien présente en contexte français.

pouvons émettre l'hypothèse, en regard de notre cadre théorique, que les enseignants français confrontés à la complexité de l'ES, mobilisent la pensée holiste, revers d'une pensée simplifiante cartésienne qu'ils maitrisent, en lieu et place de la pensée complexe qu'ils ne parviennent pas encore à conceptualiser, contrairement à leurs homologues indiens.

55 Enfin, l'intégration de l'ES dans toutes les disciplines qui définit l'approche transdisciplinaire s'accompagne également d'un questionnement, d'un sentiment de difficulté en contexte français alors que ce n'est pas le cas en contexte indien.

Pourtant, nous aurions pu nous attendre au résultat inverse puisque l'Inde a situé l'ES dans une approche disciplinaire alors que la France la situe dans une approche curriculaire (Parcours éducatif de santé). Il semble encore une fois que la conceptualisation de la pensée complexe (ici via le principe hologrammatique) soit une aide pour penser la transdisciplinarité de l'ES.

En effet, Morin (Peyron-Bonjan et Ardoino, 2000) considère qu'on ne peut pas concevoir la transversalité au sein des disciplines, sans être familier d'une pensée complexe : «On ne peut pas être transdisciplinaire sans avoir au préalable une conception de l'organisation qui permette à ce moment là de traverser les disciplines [...] on ne saurait brandir le drapeau "transdisciplinaire " sans avoir au préalable une pensée organisatrice».

La formation des enseignants français aux principes de la complexité semble donc être un pré-requis pour pouvoir appréhender sereinement et efficacement l'ES préconisée par les experts internationaux. Ainsi, dans la lignée des travaux de Santos (2011) et Descarpentries (2019) qui dénonce une épistémologie de surplomb dans le champ de la santé, s'imposant à tous les contextes indépendamment des particularismes socio- 
culturels, notre article montre qu'en contexte euro-centré (la France) ou dans un pays du Sud (l'Inde), vouloir mettre en œuvre une politique d'ES unifiée et hégémonique, est une stratégie inopérante. A la suite de Santos, nous pensons que «ce n'est pas d'alternatives dont nous avons besoin mais plutôt d'une manière alternative de penser les alternatives " (2011, p. 39); ce qui repose de façon cruciale la formation des enseignants à une épistémologie de la complexité dans le domaine de la santé.

\section{Limites de l'étude, perspectives}

Dans cette étude, nous avons travaillé avec deux échantillons d'enseignants. Les données nationales montrent que notre échantillon français est assez représentatif de la population source (au niveau national). Toutefois, notre échantillon indien est très genré (féminin). Nous avons pourtant constaté sur place une proportion un peu plus importante d'hommes dans les écoles, mais ces derniers n'ont pas souhaité participer à l'étude. Il est possible que notre propre identité de chercheuse, femme et occidentale, ait eu un impact sur la motivation des hommes indiens à accepter d'interagir avec nous lors de cette enquête. Il y aurait donc ici un léger biais d'échantillonnage.

De plus, il est difficile de généraliser à l'ensemble des enseignants indiens ce qui relève d'un seul territoire (Pondichéry) et à la France ce qui relève de l'académie de Montpellier ; mais nous pensons toutefois que notre étude lance des pistes de réflexion pour chaque contexte.

1 Notre méthodologie repose sur un test d'association d'idées. Les catégories thématiques réalisées résultent d'un travail d'interprétation consistant à identifier des champs sémantiques dans les propositions des répondants. La principale limite est liée à la polysémie des mots : les champs sémantiques que nous avons réalisés risquent de ne pas être le reflet des intentions des répondants. Bien qu'ayant fait participer trois codeurs différents pour diminuer la part de subjectivité, il convient d'interpréter ces résultats avec prudence.

2 Enfin, nous avons travaillé sur la base des représentations et pratiques déclarées. Il pourra être intéressant de compléter cette étude par une observation directe des pratiques afin d'évaluer en quoi ces pratiques d'ES intègrent effectivement les critères de complexité que nous avons étudiés.

3 En particulier, la présente étude soulève un aspect qui mériterait d'être investigué dans une recherche ultérieure. En effet, il est surprenant de constater que les enseignants indiens non pratiquant l'ES sont très enthousiastes vis-à-vis du partenariat en ayant dans le même temps assez peu de connaissances biomédicales. Ainsi, on peut se demander si cet enthousiasme est davantage lié à la possibilité de déléguer une mission pour laquelle ils ne se sentent pas suffisamment formés, qu'à la perspective de se former au contact de partenaires de santé. Une étude portant spécifiquement sur le partenariat en ES en contexte indien serait pertinente à mener ultérieurement pour clarifier cette question.

\section{Conclusion}

Notre étude a montré que la théorie de la pensée complexe est opérationnelle pour analyser la politique d'éducation à la santé actuellement préconisée par les experts 
internationaux. Elle a également montré que tous les enseignants ne sont pas également armés face au défi de cette complexité.

Si l'Inde est familière de la pensée complexe, un nombre moins important d'enseignants investissent l'éducation à la santé, comparativement à la France. Nous avons relevé que les non pratiquants indiens maitrisent peu les connaissances biomédicales nécessaires à cette éducation et identifient les professionnels de santé comme principaux acteurs de cette éducation. Nous pensons qu'un manque de formation aux contenus disciplinaires pourrait expliquer ce désinvestissement en contexte indien.

En France, la pratique de l'éducation à la santé est plus généralisée mais les enseignants expriment une perte de repère, un questionnement, une difficulté quand ils sont confronté aux trois critères de complexité de l'éducation à la santé (partenariat, approche compétences et transdisciplinarité) ; ce qui n'est pas retrouvé en contexte indien.

Pour rendre effectives les préconisations internationales déclinées à l'échelle nationale, il faudrait aider les enseignants français à sortir du "grand paradigme d'Occident » (Morin, 1990) pour rentrer dans le paradigme de complexité. Cette révolution épistémologique nécessite de repenser la formation initiale et continue des enseignants pour y intégrer une épistémologie de la complexité.

\section{BIBLIOGRAPHIE}

Alhadeff-Jones, M. (2008). Three Generations of Complexity Theories: Nuances and Ambiguities. Educational Philosophy and Theory, 40 (1): 66-82.

Arboix Calas, F. (2013). Education à la santé et complexité: proposition de formation aux stratégies nutritionnelles en milieu scolaire: le cas de la prévention globale de l'obésité dans une étude comparative franco-indienne (Doctoral dissertation, Montpellier 2).

Bardin, L. (2007). L'analyse de contenu. Paris: PUF.

Barthes, A. et Alpe, Y. (2016). Utiliser les représentations sociales en éducation. Paris : L’Harmattan.

Bizzoni-Prévieux C., Mérini C., Otis J., Jourdan D., Grenier J. (2011). Les partenariats en éducation à la santé au primaire: analyse comparée. Nouveaux cahiers de la recherche en éducation, 2(14) : 43-60.

Central Board of Secondary education CBSE (2007). School Health manual Vol.1. http://

www.schoolsanitation.com/pdf/cbse-manual-vol1.pdf

Descarpentries, J. (2019). L'éducation pour la santé durable comme levier de transformation sociale. Educations, 2(1). https://www.openscience.fr/L-education-pour-la-sante-durablecomme-levier-de-transformation-sociale

Descartes, R. (2000). Discours de la méthode (1ere Edition : 1637). Paris: Flammarion. 
Fabre, M. (2014). Les «Éducations à»: problématisation et prudence. Éducation et socialisation. Les Cahiers du CERFEE, 36. http://journals.openedition.org/edso/875 ; DOI : https://doi.org/10.4000/ edso.875

Fortin, R. (2008). Penser avec Edgar Morin. Lyon : Chronique Sociale.

Grieder, E. (s.d.) Morin, l'oriental. Réseau Canopée : Projets Pensée complexe. https:// www.reseau-canope.fr/fileadmin/user_upload/Projets/pensee_complexe/ grieder_morin_loriental.pdf

Groussin, T. et Mack, M. (2013). Une révolution pour la science : la Transdisciplinarité. Entretien avec Basarab Nicolescu. Les Cahiers de SoL (9). http://www.scenariosvision.com/S+V/Download/ SOL\%20France_Cahiers09.pdf

Jourdan, D. (2010) Education à la santé. Quelle formation à la santé pour les enseignants ? SaintDenis, INPES.

Lange, J.-M. et Victor, P. (2006). Didactique curriculaire et « éducation à... la santé, l'environnement et au développement durable »: quelles questions, quels repères? Didaskalia, 28 : 85-100.

Lenoir, Y. et Sauvé, L. (1998). Note de synthèse. De l'interdisciplinarité scolaire à l'interdisciplinarité dans la formation à l'enseignement: un état de la question. Revue française de pédagogie, 125(1), 109-146.

Mérini, C. (1999). Le partenariat en formation : de la modélisation à une application. Paris : l'Harmattan.

Ministère de l'Education nationale (2014). Repères et références statistiques. http:// cache.media.education.gouv.fr/file/2014/04/7/DEPP_RERS_2014_344047.pdf

Ministère des Solidarités et de la Santé (2017). Stratégie Nationale de Santé (2018-2022). https:// solidarites-sante.gouv.fr/IMG/pdf/dossier_sns_2017_vdef.pdf

Ministry of Human Resource dévelopment (2018). Educational Statistic at a glance. https:// mhrd.gov.in/sites/upload_files/mhrd/files/statistics-new/ESAG-2018.pdf

Morin, E. (1977). La nature de la nature. Paris : Le Seuil.

Morin, E. (1988). Le défi de la complexité. Revue Chimères, 5(6) : 1-18.

Morin, E. (1990). Introduction à la pensée complexe. Paris : Le Seuil.

Morin, E. (1994). Sur l'interdisciplinarité. Bulletin Interactif du Centre international de recherches et études transdisciplinaire, 2.

Moscovici, S. (1961). La psychanalyse, son image et son public. Paris: PUF.

Negura, L. (2006). L'analyse de contenu dans l'étude des représentations sociales. SociologieS, 1(1) : $1-21$.

O.M.S. (1993). Program on Mental Health. Life Skills Education in School. Genève.

O.M.S. (1997). L'école et son rôle dans l'éducation sanitaire et la promotion de la santé en général. Genève. Peyron-Bonjan, C. et Ardoino, J. (2000). Réforme de la pensée, pensée de la réforme: Entretiens avec Edgard Morin sur l'éducation. Pratiques de formation, (39).

Pizon, F., Jourdan, D., Simar, C., Rommel, S. et Monney, V. (2010). Les enseignants, entre quotidien et compétences en éducation pour la santé. La santé de l'Homme, 407 : 23-24

Sandrin-Berthon, B. (1997). Apprendre la santé à l'école. Montrouge : ESF Editeurs. 
Santos, B. D. S. (2011). Épistémologies du sud. Études rurales, 187, : 21-50.

UNESCO, UNICEF. WHO, World Bank, and Education International. (2002). FRESH: A Comprehensive School Health Approach to Achieve EFA. Paris.

Weaver, W. (1991). Science and complexity. In Facets of systems science (pp. 449-456). Boston : Springer.

\section{NOTES}

1. Dictionnaire Larousse en ligne

2. Central Board of Secondary Education (conseil central de l'enseignement secondaire) : il est le principal organe directeur de l'enseignement secondaire en Inde. Il a en charge l'organisation des examens et s'occupe du fonctionnement de toutes les écoles primaires accréditées.

\section{RÉSUMÉS}

A l'instar des « éducations à ", l'éducation à la santé porte plusieurs " pièges épistémologiques " potentiels qui suscitent perplexité et perte de repère chez les enseignants français. Cet article propose d'analyser la nature de ces pièges potentiels par le prisme d'une approche de la complexité. Nous montrons que l'éducation à la santé actuellement préconisée par les experts internationaux est construite sur trois piliers qui illustrent les principes d'une pensée complexe. A travers une étude empirique, nous identifions quelques freins et leviers à la mise en œuvre de cette éducation dans un contexte indien, imprégné par un paradigme de complexité et dans un contexte français, imprégné par un paradigme de pensée simplifiante. Ces éléments interrogent la pertinence de donner à l'éducation à la santé, une dimension transculturelle.

As other forms of education, health education contains several potential "epistemological traps" which cause perplexity and loss of reference among French teachers. This article propose to analyze the nature of these potential traps through the prism of an approach of complexity. We show that the health education currently advocated by international experts is built on three pillars that illustrate the principles of complex thinking. Through an empirical study, we identify some obstacles and facilitators to the implementation of this education in an Indian context, steeped in a paradigm of complexity and in a French context, steeped in a paradigm of simplification. These elements question the relevance of giving to health education, a crosscultural dimension.

\section{INDEX}

Keywords : health éducation, complexity, représentations, practices, obstacles

Mots-clés : éducation à la santé, complexité, représentations, pratiques, obstacles 
AUTEUR

FRANCE ARBOIX

Université de Montpellier, Laboratoire Interdisciplinaire de Recherche en Didactique, Education

et Formation 